ARTICLE

DOI: $10.1038 / s 41467-018-06359-y$

OPEN

\title{
Sounding-rocket microgravity experiments on alumina dust
}

\author{
Shinnosuke Ishizuka (1) 1, Yuki Kimura (1) ${ }^{1}$, Itsuki Sakon², Hiroshi Kimura ${ }^{3}$, Tomoya Yamazaki (1) ${ }^{1}$ \\ Shinsuke Takeuchi ${ }^{4,5}$ \& Yuko Inatomi (10) 4,5
}

Alumina $\left(\mathrm{Al}_{2} \mathrm{O}_{3}\right)$ is believed to be the first major condensate to form in the gas outflow from oxygen-rich evolved stars because of the refractoriness and that $\alpha-\mathrm{Al}_{2} \mathrm{O}_{3}$ (corundum, most stable polymorph) is a potential origin of a $13 \mu \mathrm{m}$ feature that appears close to stars. However, no one has directly reproduced the $13 \mu \mathrm{m}$ feature experimentally, and it has remained as a noteworthy unidentified infrared band. Here, we report nucleation experiments on $\mathrm{Al}_{2} \mathrm{O}_{3}$ nanoparticles monitored by a specially designed infrared spectrometer in the microgravity environment of a sounding rocket. The conditions approximate to those around asymptotic giant branch (AGB) stars. The measured spectra of the nucleated $\mathrm{Al}_{2} \mathrm{O}_{3}$ show a sharp feature at a wavelength of $13.55 \mu \mathrm{m}$ and comparable in width to that observed near oxygen-rich $A G B$ stars. Our finding that $\alpha-\mathrm{Al}_{2} \mathrm{O}_{3}$ nucleates under certain condition provides a solid basis to elaborate condensation models of dust around oxygen-rich evolved stars.

\footnotetext{
${ }^{1}$ Institute of Low Temperature Science, Hokkaido University, Hokkaido, Sapporo 060-0819, Japan. ${ }^{2}$ Department of Astronomy, School of Science, University of Tokyo, 7-3-1 Hongo, Bunkyo-ku, Tokyo 113-0033, Japan. ${ }^{3}$ Planetary Exploration Research Center (PERC), Chiba Institute of Technology, Tsudanuma 2-17-1, Narashino, Chiba 275-0016, Japan. ${ }^{4}$ Institute of Space and Astronautical Science, Japan Aerospace Exploration Agency, 3-1-1 Yoshinodai, Chuo-ku, Kanagawa, Sagamihara 229-8510, Japan. ${ }^{5}$ School of Physical Sciences, SOKENDAl (The Graduate University for Advanced Studies), 3-1-1 Yoshinodai, Chuo-ku, Kanagawa, Sagamihara 252-5210, Japan. Correspondence and requests for materials should be addressed to

Y.K. (email: ykimura@lowtem.hokudai.ac.jp)
} 
symptotic giant branch (AGB) stars, a type of evolved stars, are significant sources of the dust present in interstellar space. Advances in interferometric observations of low-mass stars, such as AGB stars in their late evolution stages, have demonstrated the existence of shells consisting of relatively large $(\sim 300 \mathrm{~nm})$ grains close (within two stellar radii) to the stars $^{1}$. Therefore, the dust must be refractory to condense, and abundant to be detected by observations. Recently, the Atacama Large Millimeter Array (ALMA) visualized the spatial distribution of molecular rotational bands around the alumina $\left(\mathrm{Al}_{2} \mathrm{O}_{3}\right)$ rich AGB star W Hya demonstrating that $\mathrm{AlO}$ gases are consumed within the dust formation region while $\mathrm{SiO}$ molecules remain gaseous even at the outside of the dust formation region ${ }^{2}$. Further, the presence of titania $\left(\mathrm{TiO}_{2}\right)$ in the gas phase far from the dust shell is reported for a red supergiant environment ${ }^{3}$. Although several minerals, such as $\mathrm{Al}_{2} \mathrm{O}_{3}, \mathrm{TiO}_{2}$, and spinel $\left(\mathrm{MgAl}_{2} \mathrm{O}_{4}\right)$, have been proposed as carriers of the $13 \mu \mathrm{m}$ feature ${ }^{4}$, $\mathrm{Al}_{2} \mathrm{O}_{3}$ is the most likely mineral to be capable of surviving sublimation and, owing to its transparency, of withstanding the radiation pressure from the central star; furthermore, it is considered to condense earlier than other abundant minerals, such as silicates. The discovery of micron-sized presolar $\mathrm{Al}_{2} \mathrm{O}_{3}$ dust with an unusual oxygen isotopic composition in meteorites strongly suggests that some of the $\mathrm{Al}_{2} \mathrm{O}_{3}$ dust has been formed around AGB stars. e.g. ref. ${ }^{5}$.

Alumina exhibits a number of crystalline structures (e.g., $\alpha, \theta$, $\delta$, or $\gamma$ ); i.e., it can exist in a diversity of polymorphic forms. Significant differences in the oxygen anion alignment in hexagonal closed-packed $\alpha-\mathrm{Al}_{2} \mathrm{O}_{3}$ (corundum) and in face-centered cubic $\gamma^{-}, \delta$-, or $\theta-\mathrm{Al}_{2} \mathrm{O}_{3}$ lead to distinct differences in the corresponding infrared (IR) band structures ${ }^{6}$. Amorphous and $\gamma$ $\mathrm{Al}_{2} \mathrm{O}_{3}$ have been considered to contribute to the $10-20 \mu \mathrm{m}$ dust extinction around red supergiant stars and $\mathrm{AGB}$ stars ${ }^{7-9}$. In addition, $\alpha-\mathrm{Al}_{2} \mathrm{O}_{3}$, the form of $\mathrm{Al}_{2} \mathrm{O}_{3}$ that is stable from $0 \mathrm{~K}$ to the melting point in the bulk size, has been proposed as a candidate for the origin of the unidentified $13 \mu \mathrm{m}$ feature that has been observed for AGB stars with low mass-loss rates ${ }^{10}$. The IR features in the spectrum of mineral particles have been calculated by using optical constants obtained for the corresponding large single crystals, based on Mie theories. The features vary with the size distribution ${ }^{11}$, temperature ${ }^{12}$, and shape formed in anisotropic crystal growth ${ }^{13}$. The spectral profiles have been successfully reproduced by $\mathrm{Al}_{2} \mathrm{O}_{3}$ with an appropriate choice of these parameters.

However, the $13 \mu \mathrm{m}$ feature has not been reproduced experimentally. The average feature observed for the unidentified $13 \mu \mathrm{m}$ band has a full width at half maximum (FWHM) of $0.5-0.8^{13}$, $0.6^{14}$, or $1.1 \mu \mathrm{m}^{15}$. In contrast to the narrow feature observed astronomically, spectra measured on powders prepared in the laboratory have shown a markedly greater width $(\Delta \lambda=6.0 \mu \mathrm{m})$. This is also true for measurements of the IR spectrum of $\alpha-\mathrm{Al}_{2} \mathrm{O}_{3}$ particles in a free-flying state in a medium with a dielectric constant that approximates to that of the astronomical environment $^{16}$. In addition to the size distribution, shape, and anisotropy, the electromagnetic interactions between particles induced during sample preparation broaden the peak ${ }^{17,18}$. These effects prevent a direct comparison with astronomical observations.

We performed in situ IR measurements on free-flying $\mathrm{Al}_{2} \mathrm{O}_{3}$ in condensation experiments involving homogeneous nucleation in a ground-based laboratory; an IR spectrum of monodispersed $\mathrm{Al}_{2} \mathrm{O}_{3}$ particles of uniform size and shape was successfully obtained ${ }^{19}$. However, an $\alpha-\mathrm{Al}_{2} \mathrm{O}_{3}$ phase has never been directly synthesized by nucleation and growth processes in the vapor phase $^{19}$. We believe the reason for this is the rapid cooling of the nascent $\mathrm{Al}_{2} \mathrm{O}_{3}$ particles by the thermal convection of the argon (Ar) buffer gas used to reduce the mean free path of the evaporated vapor, and thereby to reduce the physical scale of the experimental system. In the ground-based experiment, a strong convection $\left(10-15 \mathrm{~cm} \mathrm{~s}^{-1}\right)$ of Ar gas is induced by the hot evaporation source. By contrast, in a microgravitational environment, this thermal convection is completely suppressed, allowing the evaporated gases to cool more slowly. Consequently, the $\mathrm{Al}_{2} \mathrm{O}_{3}$ nuclei have higher possibility to overcome a large barrier to form the most stable corundum phase. In addition, the slower cooling permits the gaseous atoms to collide more frequently and, on a longer timescale of gas cooling, provides a more realistic simulation of the grain formation in gas outflows from AGB $\operatorname{stars}^{20}$. Based on this concept, we performed an idealnucleation experiment in a microgravitational environment of $2.2 \times 10^{-3} \mathrm{~m} \mathrm{~s}^{-2}\left(2.2 \times 10^{-4} \mathrm{G}\right)$ aboard the sounding rocket S-520 30 and successfully reproduce a $13 \mu \mathrm{m}$ band with a very narrow width of $0.5-0.6 \mu \mathrm{m}$. The experimental method will provide sufficient data to permit the identification of the unidentified stellar IR bands.

\section{Results}

Formation of $\mathrm{Al}_{2} \mathrm{O}_{3}$ in a microgravitational environment. To measure the temporal evolution of the IR spectra of free-flying $\mathrm{Al}_{2} \mathrm{O}_{3}$ nanoparticles nucleated in the experimental chamber in a microgravitational environment, we have designed and fabricated an IR grating spectrometer system to meet the size limitations of the sounding rocket S-520, and endure impacts of the launch (Fig. 1 and Methods). The experimental chamber entered a microgravitational environment $50 \mathrm{~s}$ after the rocket launch, as confirmed by the acceleration measurements (Supplementary Fig. 1). Ninety seconds after the launch, the aluminum (Al) evaporation source was electrically heated on a tantalum $(\mathrm{Ta})$ filament in a gas mixture of $\operatorname{Ar}\left(3.8 \times 10^{4} \mathrm{~Pa}\right)$ and oxygen $(2.0 \times$ $\left.10^{3} \mathrm{~Pa}\right)$. The evaporated $\mathrm{Al}$ reacted with the oxygen to form $\mathrm{Al}_{2} \mathrm{O}_{3}$ nanoparticles (Methods). The total pressure rose and then fell due to heating and cooling of the evaporation source, respectively (Supplementary Fig. 2). After the experiment, the total pressure was by $1700 \mathrm{~Pa}$ lower than before the experiment. Evidently, oxygen was consumed by the $\mathrm{Al}$ oxidation to form $\mathrm{Al}_{2} \mathrm{O}_{3}$ nanoparticles.

Measurement of an infrared spectra with a narrow band. The raw spectra obtained for $10 \mathrm{~Hz}$ were divided into several series based on variations in the spectral shape during the experiment (Supplementary Fig. 3). We averaged 32 spectra recorded before the evaporation of $\mathrm{Al}_{2} \mathrm{O}_{3}$ in the $92.4-96.5 \mathrm{~s}$ after the launch to provide a background spectrum (Supplementary Fig. 3; BG). In the 97.6-100.2 $\mathrm{s}$ after the launch, exactly after the evaporation, a swelling appeared, extending from $11 \mu \mathrm{m}$ toward longer wavelengths, with a maximum at $12-13 \mu \mathrm{m}$ (Fig. 2a). At 100.2-103.0 s after the launch, the IR spectra discontinuously fluctuated with time, because hot fragments of the evaporation source drifted into the optical path (Supplementary Fig. 4). At $103.0 \mathrm{~s}$ after launch, the IR spectra stabilized (Fig. 2b) and intensified, retaining the shape of the spectra measured 97.6-100.2 s after the launch. Approximately $2.7 \mathrm{~s}$ after the evaporation, a sharp absorption at $13.55 \pm 0.05 \mu \mathrm{m}$ began to intensify (Fig. $2 \mathrm{c}$ shows the spectrum at 105.6-109.7 $\mathrm{s}$ after the launch). This absorption continued for several tens of seconds without showing any change in the band shape (Fig. 2d). The FWHM of the sharp $13.55 \pm 0.05 \mu \mathrm{m}$ band was $0.55 \pm 0.05 \mu \mathrm{m}$ after subtraction of the baseline. This is the first experimental evidence that the IR band arising from $\mathrm{Al}_{2} \mathrm{O}_{3}$ nanoparticles has a bandwidth consistent with theoretical predictions (Supplementary Figs. 5-9) and the unidentified $13 \mu \mathrm{m}$ band observed around oxygen-rich AGB stars (Fig. 3b). 


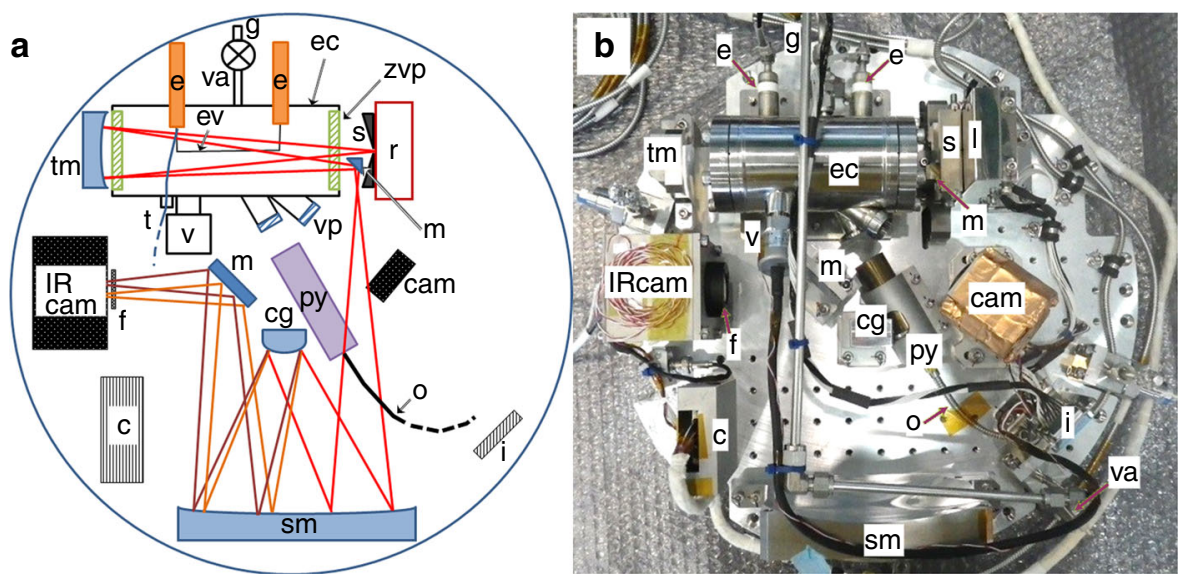

Fig. 1 The optical system of the dispersive IR spectrometer with the experimental chamber. a Schematic representation; b corresponding photograph. The IR radiation (magenta lines) was dispersed at the convex grating (cg). The resulting intensity of the IR radiation was recorded with an IR camera (IR cam). The evaporation source (ev), consisting of an Al wire wrapped around a Ta filament $(0.2 \mathrm{~mm}$ in diameter and $67 \mathrm{~mm}$ in length) is depicted by the black solid line in the experimental chamber (ec). The other labels are as follows: (c) IR camera controller; (e) electrode; (f) IR filter; ( $g$ ) gas line; (i) interface connecters; (m) mirror; (o) optical fiber; ( $r$ IR radiation source; (s) slit; (t) thermocouple; (v) vacuum gauge; (cam) CMOS camera; (py) pyrometer; (sm) spherical mirror; (tm) toroidal mirror; (va) valve; (vp) view port; (zvp) ZnSe view port. The evacuation of the air and subsequent injection of Ar gas into the chamber were performed on the ground after the experimental system had been installed inside the rocket

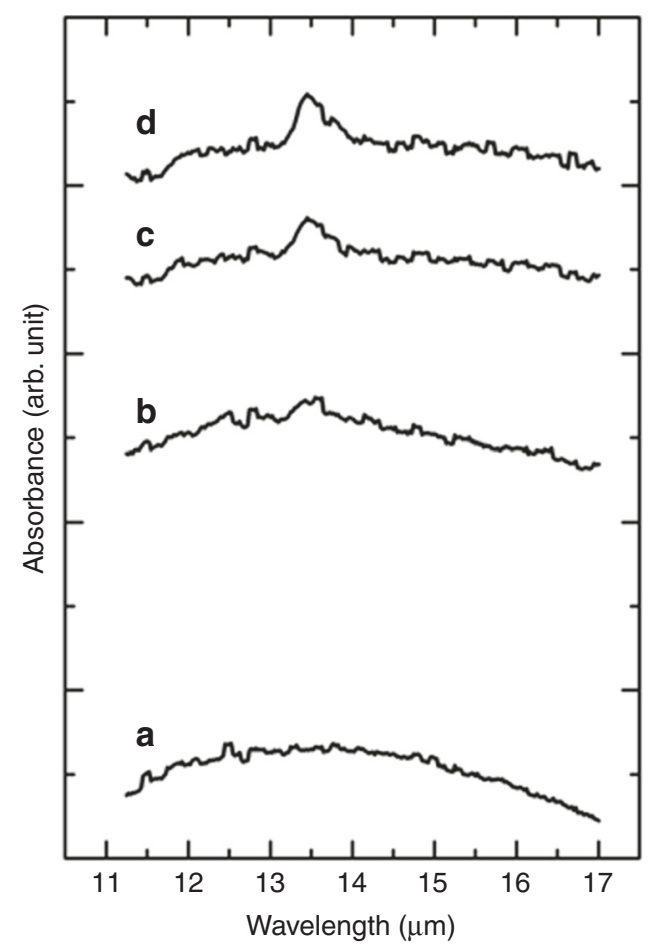

Fig. 2 Absorption spectra of nascent $\mathrm{Al}_{2} \mathrm{O}_{3}$ nanoparticles. These spectra have been accumulated at (a) 97.6-100.2, (b) 103.0-104.5, and (c) 105.6-109.7 s after the launch; the background spectrum BG (Supplementary Fig. 3) was subtracted and the spectra were not offset. The $13.55 \pm 0.05 \mu \mathrm{m}$ absorbance that appeared in c remained for a long period. The stationary absorbance at 109.9-114.0 s after the launch is shown in $\mathbf{d}$

\section{Discussion}

The IR spectra of the free-flying nanoparticles can be directly compared with astronomical observations, because the dielectric constant of the surrounding gas is similar to that of vacuum (Fig. 3) $19,21-23$. Nanoparticles formed in microgravitational environments will have a greater degree of homogeneity in terms

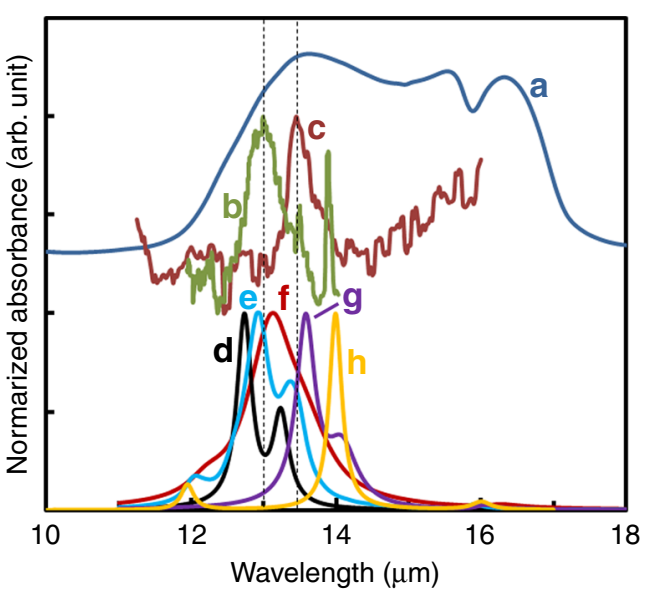

Fig. 3 Comparison of IR spectra. These spectra were obtained: a with laboratory measurements on $\alpha-\mathrm{Al}_{2} \mathrm{O}_{3}$ embedded in a $\mathrm{KBr}$ medium (blue) ${ }^{23}$; b by observation of $A G B$ stars (khaki) ${ }^{4}$; c in this study (brown). Calculated spectra of d: spheres at $300 \mathrm{~K}$ (black); e faceted particle at $300 \mathrm{~K}$ (light blue); $\mathbf{f}$ spheres at $928 \mathrm{~K}$ (magenta); $\mathbf{g}$ spheres with surface contamination of Ta of $5 \mathrm{~nm}$ in thickness (purple); $\mathbf{h}$ oblate flattened along c-axis with ratio of 0.3 (yellow)

of their size, shape, and crystallinity, because they nucleate and grow in environments with a low Reynolds number, which prevents fluctuations in local concentrations of the evaporated vapor through turbulences ${ }^{24}$. Such homogeneous environments permit gases to undergo extreme cooling, thereby increasing the supersaturation ratio significantly and permitting the formation of nanoparticles of uniform size and shape. The homogeneity of particles generally reduces the bandwidths of their spectra. The previously reported IR spectra of free-flying particles have shown complex shapes and distributions ${ }^{16}$.

The metastable phases $\gamma$-, $\delta$-, and $\theta-\mathrm{Al}_{2} \mathrm{O}_{3}$, which have been formed in ground-based experiments, have peaks centered at 12 $\mu \mathrm{m}$ with strong satellite peaks in the range of $10-20 \mu \mathrm{m}$ showing quite different features than those in the spectrum ${ }^{16,19,25}$. The formation of these metastable phases is inconsistent with the 
results because of the lacking of $12 \mu \mathrm{m}$ band. Even the other $\mathrm{Al}_{2} \mathrm{O}_{3}$ polymorphs, such as $\kappa$ and $\chi$, which are usually formed in the dehydration process of hydrous precursors, accompanied much broad and complex absorption bands in the whole $10-20 \mu \mathrm{m}$ range ${ }^{14,22}$. Therefore, the most stable phase, $\alpha-\mathrm{Al}_{2} \mathrm{O}_{3}$, is the only possible candidate to reproduce the IR absorption feature at $13.55 \pm 0.05 \mu \mathrm{m}$ (Supplementary Note 1). We calculated the $13 \mu \mathrm{m}$ feature assuming the properties (facet (Supplementary Fig. 5), temperature (Supplementary Fig. 6), surface contamination (Supplementary Fig. 7), anisotropy (Supplementary Fig. 8), and coagulation (Supplementary Fig. 9)) of $\alpha-\mathrm{Al}_{2} \mathrm{O}_{3}$ by using the Mie theory and the discrete-dipole approximation (Supplementary Note 2). This difference in the peak position in our spectra from that of the astronomically observed $13 \mu \mathrm{m}$ band, which typically occurs at $12.5-13.0 \mu \mathrm{m}^{2}$, is possibly a combination of the result of an edge effect of the particle and contamination on their surface in addition to differences in temperature and/or anisotropy of the $\alpha-\mathrm{Al}_{2} \mathrm{O}_{3}$ nanoparticles (Supplementary Note 3 ). In our groundbased experiments, the condensed $\theta-\mathrm{Al}_{2} \mathrm{O}_{3}$ nanoparticles were faceted with edges and occasionally contaminated with amorphous $\mathrm{Al}_{2} \mathrm{O}_{3}$ and $\mathrm{Ta}$ when the Ta filament burned out (Supplementary Note 1$)^{19}$. The $\alpha-\mathrm{Al}_{2} \mathrm{O}_{3}$ nanoparticles produced in the current experiment might have also been faceted with sharp edges, which would shift their peak position toward a longer wavelength (Fig. 3e and Supplementary Fig. 5). This confirms that if the origin of the $13 \mu \mathrm{m}$ band is the $\alpha-\mathrm{Al}_{2} \mathrm{O}_{3}$, the dust particles should have no thick contamination, have approximately a spherical shape, and should rarely aggregate together to maintain a narrow bandwidth. In contrast to the grown particles in this experiment, natural $\alpha-\mathrm{Al}_{2} \mathrm{O}_{3}$ particles in gas outflows of evolved stars will have experienced evaporation and/or sputtering, including incorporation of other elements, and will consequently have round edges. We believe that the differences in the peak wavelength between the experimental measurements and astronomical observations provide us with additional details regarding the condensation processes in AGB stars.

In this study, a significant delay existed between the typical timescale for gas cooling $\left(\sim 10^{-3} \mathrm{~s}\right)$ after the termination of the evaporation (101.4 s after the launch) and the timescale of $2.7 \mathrm{~s}$ for the appearance of the sharp $13.55 \pm 0.05 \mu \mathrm{m}$ band. $\mathrm{An}^{\mathrm{Al}_{2} \mathrm{O}_{3}}$ nucleation experiment performed under the same gas atmosphere on the ground resulted in the formation of $\delta-\mathrm{Al}_{2} \mathrm{O}_{3}$ with a twostep process. In the first step, liquid-like particles that showed a broad absorption extending from $11 \mu \mathrm{m}$ to longer wavelengths nucleated from the supersaturated vapor at a temperature considerably below the melting point of bulk $\mathrm{Al}_{2} \mathrm{O}_{3}$. In the second step, the crystallization to $\mathrm{Al}_{2} \mathrm{O}_{3}$ was induced in the liquid-like particles while the nanoparticles were freely flying ${ }^{19}$. The $2.7 \mathrm{~s}$ delay observed in this study suggests that the liquid-like particles nucleated from the gas, and then the $\alpha-\mathrm{Al}_{2} \mathrm{O}_{3}$ nucleated in these in a similar manner to that in the ground-based experiment. Later, a broad swell appeared with the sharp $13.55 \pm 0.05 \mu \mathrm{m}$ band. The shape of the broad swell in Fig. $2 \mathrm{~b}$ is consistent with the presence of noncrystalline $\mathrm{Al}_{2} \mathrm{O}_{3}{ }^{6}$. However, the identification is not certain because the absorption covers approximately the complete measurement range. With an increasing surface area-tovolume ratio, a stability crossover from $\alpha-\mathrm{Al}_{2} \mathrm{O}_{3}$ to $\gamma-\mathrm{Al}_{2} \mathrm{O}_{3}$ and from $\gamma-\mathrm{Al}_{2} \mathrm{O}_{3}$ to amorphous $\mathrm{Al}_{2} \mathrm{O}_{3}$ occurs for surface areas exceeding 75 and $370 \mathrm{~m}^{2} \mathrm{~g}^{-1}$, respectively, which correspond to the sizes of spheres with diameters of $\sim 20$ and $\sim 5 \mathrm{~nm}$, respectively $y^{26,27}$. In the atmosphere of AGB stars, the size of newly nucleated $\mathrm{Al}_{2} \mathrm{O}_{3}$ particles should be smaller than these sizes because the process began with the agglomeration of molecules (as in the present experiment).

A neutron diffraction study of an $\mathrm{Al}_{2} \mathrm{O}_{3}$ melt kept in a freeflying state at $2500 \mathrm{~K}$ showed that $62 \%$ of $\mathrm{Al}$ was present as $\mathrm{AlO}_{4}$,
$24 \%$ as $\mathrm{AlO}_{5}$, and $2 \%$ as $\mathrm{AlO}_{6}{ }^{28}$. Numerical studies have suggested that a large proportion of $\mathrm{Al}$ adopts the $\mathrm{AlO}_{4}$ tetrahedral form in the melt ${ }^{29,30}$. The metastable forms of $\mathrm{Al}_{2} \mathrm{O}_{3}\left(\gamma-\mathrm{Al}_{2} \mathrm{O}_{3}, \delta\right.$ $\mathrm{Al}_{2} \mathrm{O}_{3}$, and $\theta-\mathrm{Al}_{2} \mathrm{O}_{3}$ ), which consist of $\mathrm{AlO}_{4}$ tetrahedra ${ }^{6,31,32}$, have (owing to their higher concentration of growth units) a higher chance of nucleation than $a-\mathrm{Al}_{2} \mathrm{O}_{3}$, which is composed exclusively of $\mathrm{AlO}_{6}$ octahedra. To overcome the larger activation energy required for the nucleation of $\alpha-\mathrm{Al}_{2} \mathrm{O}_{3}$, a higher temperature might be required. Under microgravity conditions, the liquid-like particles might have remained at higher temperatures for a longer time than those on the ground. At a gas pressure of $4.0 \times 10^{4} \mathrm{~Pa}$, the temperature of the nanoparticles is almost identical to that of the surrounding gas ${ }^{19}$. Owing to the reported temperature field around the evaporation source at $2226 \pm 22 \mathrm{~K}$ under an $\mathrm{Ar}$ atmosphere of $4.0 \times 10^{4} \mathrm{~Pa}^{20}$, the nascent nucleated nanoparticles can retain a temperature of about $1000 \mathrm{~K}$ for a few seconds. This relatively long incubation time for the nucleation can induce the formation of $\alpha-\mathrm{Al}_{2} \mathrm{O}_{3}$ within the liquid-like particles under microgravity conditions.

The agreement regarding the width of the $13 \mu \mathrm{m}$ feature observed in this study increases the evidence for the existence of $\alpha$ $\mathrm{Al}_{2} \mathrm{O}_{3}$ around AGB stars. We expect that future observations at a higher spatial resolution with the James Webb Space Telescope ${ }^{33}$ will confirm the transition from noncrystalline $\mathrm{Al}_{2} \mathrm{O}_{3}$, which shows a broad feature at $11-18 \mu \mathrm{m}$, to $\alpha-\mathrm{Al}_{2} \mathrm{O}_{3}$, which shows a sharp $13 \mu \mathrm{m}$ feature. The spectral region from 10 to $40 \mu \mathrm{m}$ corresponds to the vibrational modes of various mineral types (e.g., crystalline olivine, crystalline pyroxene, or magnesium sulfide). Therefore, comparing the IR features obtained by astronomical observations with the experimental dataset is crucial to identify the origin of $\mathrm{dust}^{34}$. The future observations with the Space Infrared Telescope for Cosmology and Astrophysics (SPICA) ${ }^{35}$ together with microgravity experiments are expected to provide a better understanding of the material evolution in the universe.

\section{Methods}

In situ IR measurement system. We have previously developed a technique to perform in situ IR measurements during the nucleation and growth of nanoparticles with a gas evaporation method in ground-based experiments ${ }^{18,21,22}$. By using a similar technique, we studied the IR spectra of free-flying nucleating $\mathrm{Al}_{2} \mathrm{O}_{3}$ nanoparticles in a microgravitational environment. The experimental apparatus had to withstand the shock generated during the launch of the sounding rocket and had to fit into the limited space available inside the rocket. Therefore, we specially designed an experimental apparatus equipped with a dispersive IR spectrometer (Fig. 1 and Supplementary Fig. 1). The IR radiation from a SiC source (IRH-1; Siliconit Corp.) passed through the experimental chamber consisting of a $150-\mathrm{mm}$ long stainless-steel cylinder with an internal diameter of $\sim 65 \mathrm{~mm}$. The radiation was then reflected by a toroidal mirror and passed through the experimental chamber once more to increase the column density. The windows of the experimental chamber were made of $\mathrm{ZnSe}$ with an anti-reflection coating; this material has a high transmittance in the $9-17 \mu \mathrm{m}$ band. The reflected radiation in the Offner-type spectrometer formed an image of the IR spectrum on the detector array $(320 \times 240$ pixels with a pixel pitch of $\delta x=23.5 \mu \mathrm{m}$ per pixel $)$ of an IR camera (C200C; Nippon Avionics Co., Ltd.) after the dispersion of the wavelength via the convex grating for $>300$ pixels in the horizontal direction. The signal intensity of the light at each pixel on the array was accumulated at the 240 pixels on the vertical axis. As a result, a single-beam spectrum was obtained at $10 \mathrm{~Hz}$. The wavelength resolution $R=\lambda / \Delta \lambda$ was 70-101 (Supplementary Fig. 6) with

$$
\Delta \lambda=\left\{(\delta \lambda \times W)^{2}+(\delta \lambda \times \Delta \mathrm{PSF})^{2}\right\}^{0.5},
$$

where $\delta \lambda$ is the pixel linear dispersion $(0.027 \mu \mathrm{m}$ per pixel), $W$ is the slit width ( $94 \mu \mathrm{m}$, corresponding to four pixels on the array detector), and $\triangle \mathrm{PSF}$ is the performance of the sensor focus. The value of $\triangle$ PSF can be expressed as follows:

$$
\Delta \mathrm{PSF}=1.22 \times \lambda \times F \times \delta x^{-1},
$$

where $F$ is the $f$-number (5.5) of the camera system. We measured the IR spectrum of a polystyrene film as a standard by using the dispersive IR spectrometer and a Fourier-transform IR spectrometer (Spectrum 400; PerkinElmer, Waltham, MA). The absolute wavelength was determined using the central wavelength of the FWHM of the strong absorption of the standard polystyrene at $13.72 \mu \mathrm{m}$. 
Preparation of the nucleation chamber. The chamber was evacuated to a pressure of $<1 \times 10^{-4} \mathrm{~Pa}$ through a quarter-inch stainless-steel tube (Fig. 1g) connected to a vacuum system composed of a turbomolecular pump (TG50F, $50 \mathrm{~L} \mathrm{~s}^{-1}$; Osaka Vacuum, Ltd.) and a scroll-type dry vacuum pump (DIS-90; ULVAC Kiko Inc., Saito City). Three days before the launch, high-purity $\mathrm{O}_{2}(99.9 \%)$ and $\operatorname{Ar}(>99.9999 \%)$ were injected into the experimental chamber. Initially, the pressure was raised to $2 \times 10^{3} \mathrm{~Pa}$ with $\mathrm{O}_{2}$ gas and then raised to a total pressure of $4.0 \times 10^{4}$ $\mathrm{Pa}$ with Ar. The gas pressure was measured with a capacitance manometer (CCMT-1000D; ULVAC KIKO Inc.) at a position just outside the valve (Fig. 1g). After the valve was closed, the gas pressure in the experimental chamber was monitored by using a specially coordinated high-resolution pressure gauge (HAV-60KP-V; Sensez Co., Tokyo) to confirm that the pressure was maintained at the time of the experiment. The accuracy of the pressure measurement was $\pm 90 \mathrm{~Pa}$.

Evaporation source of alumina. Al metal wire $(\phi=0.1 \mathrm{~mm}$, purity $99.99 \%$; Nilaco Corp.) was coiled around a Ta filament ( $\phi=0.2 \mathrm{~mm}$, purity $99.95 \%$; Nilaco Corp.), and the assembly was positioned in the experimental chamber as an evaporation source with its axis aligned to the optical axis. The evaporation source was connected to $\mathrm{Cu}$ electrodes (PF-SM6-3KV-10A; Kawaso Texcel Co., Osaka) to permit rapid electrical-resistance heating. The evaporation source was positioned $21 \mathrm{~mm}$ below the center of the optical path to reduce emission noise during heating.

Procedures after the launch. The S-520 30 sounding rocket of the Japan Aerospace Exploration Agency (JAXA), which carried the apparatus to an altitude of $312 \mathrm{~km}$, was launched at 8:00 pm JST on 11 September 2015 from the Uchinoura Space Center, Japan. Approximately $50 \mathrm{~s}$ after the launch, the rocket entered microgravity. Then, $90 \mathrm{~s}$ after the launch, a dc voltage of $1.0 \mathrm{~V}$ was applied to the evaporation source; the voltage was then increased at a constant rate of $\sim 0.5 \mathrm{~V} \mathrm{~s}^{-1}$. The temperature of the evaporation source was measured using a pyrometer (ISQ5-LO; LumaSense Technologies, Inc., USA) and K-type thermocouples with diameters of $0.1 \mathrm{~mm}$ (a combination of WF-1/8"PT-0.8-2-T-TK- $1000 \mathrm{~mm} / 150 \mathrm{~mm}$ (Tecsam Co., Ltd., Hsinchu) and KMT-100-100-050 (ANBE SMT Co., Yokohama)) set at the base of the Ta wire. The electrical-resistance heating led to the $\mathrm{Al}$ evaporation, and nanoparticles formed from the cooling of evaporated particles. At $101 \mathrm{~s}$ after the launch, the evaporation source burned out (Supplementary Fig. 4). The heating of the evaporation source was confirmed with a CMOS camera (MS-M33WT3; MOSWELL Co., Ltd., Yokohama). The recorded data from the flying rocket were received by the telemetry equipment at the Uchinoura Space Center, Japan.

\section{Data availability}

The datasets generated during and/or analyzed during the current study are available from the corresponding author on reasonable request.

Received: 19 July 2018 Accepted: 20 August 2018

Published online: 19 September 2018

\section{References}

1. Norris, B. R. M. et al. A close halo of large transparent grains around extreme red giant stars. Nature 484, 220-222 (2012).

2. Takigawa, A., Kamizuka, T., Tachibana, S. \& Yamamura, I. Dust formation and wind acceleration around the aluminum oxide-rich AGB star W Hydrae. Sci. Adv. 3, eaao2149 (2017).

3. De Beck, E. et al. ALMA observations of $\mathrm{TiO}_{2}$ around VY CMa. J. Phys. Conf. Ser. 728, 022009 (2016).

4. Posch, T. et al. On the origin of the $13 \mu \mathrm{m}$ feature: a study of ISO-SWS spectra of oxygen-rich AGB stars. Astron. Astrophys. 352, 609-618 (1999).

5. Stroud, R. M., Nittler, L. R. \& Alexander, C. M. O. 'D. Polymorphism in presolar $\mathrm{Al}_{2} \mathrm{O}_{3}$. Science 305, 1455-1457 (2004).

6. Levin, I. \& Brandon, D. Metastable alumina polymorphs: crystal structures and transition sequences. J. Am. Ceram. Soc. 81, 1995-2012 (1998).

7. Onaka, T., de Jong, T. \& Willems, F. A study of M Mira variables based on IRAS LRS observations II. Model fits and derived parameters for 109 Miras. Astron. Astrophys. Suppl. Ser. 81, 261-284 (1989).

8. Onaka, T., de Jong, T. \& Willems, F. J. A study of M Mira variables based on IRAS LRS observations. I. Dust formation in the circumstellar shell. Astron. Astrophys. 218, 169-179 (1989)

9. Miyata, T. et al. A spectroscopic study of dust around 18 oxygen-rich Mira variables in the N band. I. Dust profiles. Astrophys. J. 531, 917-927 (2000).

10. Kwok, S. The synthesis of organic and inorganic compounds in evolved stars. Nature 430, 985-991 (2004).
11. DePew, K., Speck, A. \& Dijkstra, C. Astromineralogy of the $13 \mu \mathrm{m}$ feature in the spectra of oxygen-rich asymptotic giant branch stars. I. Corundum and spinel. Astrophys. J. 640, 971-981 (2006).

12. Zeidler, S., Posch, Th \& Mutschke, H. Optical constants of refractory oxides at high temperatures: mid-infrared properties of corundum, spinel, and $\alpha$-quartz, potential carriers of the $13 \mu \mathrm{m}$ feature. Astron. Astrophys. 553, A81 (2013).

13. Takigawa, A., Tachibana, S., Nagahara, H. \& Ozawa, K. Evaporation and condensation kinetics of corundum: the origin of the $13 \mu \mathrm{m}$ feature of oxygenrich AGB stars. Astrophys. J. Suppl. Ser. 218, 2 (2015).

14. Sloan, G. C., LeVan, P. D. \& Little-Marenin, I. R. Sources of the 13 micron feature associated with oxygen-rich circumstellar dust. Astrophys. J. 463, 310-319 (1996).

15. Begemann, B. et al. Aluminum oxide and the opacity of oxygen-rich circumstellar dust in the 12-17 micron range. Astrophys. J. 476, 199-208 (1997).

16. Tamanai, A. et al. Morphological effects on IR band profiles: experimental spectroscopic analysis with application to observed spectra of oxygen-rich AGB stars. Astron. Astrophys. 501, 251-267 (2009).

17. Bohren, C. F. \& Huffman, D. R. Absorption and Scattering of Light by Small Particles (Wiley, New York, 1983).

18. Mutschke, H., Min, M. \& Tamanai, A. Laboratory-based grain-shape models for simulating dust infrared spectra. Astron. Astrophys. 504, 875-882 (2009).

19. Ishizuka et al. Two-step process in homogeneous nucleation of alumina in supersaturated vapor. Chem. Mater. 28, 8732-8741 (2016).

20. Kimura, Y. et al. Pure iron grains are rare in the universe. Sci. Adv. 3, e1601992 (2017).

21. Tamanai, A., Mutschke, H., Blum, J. \& Neuhäuser, R. Experimental infrared spectroscopic measurement of light extinction for agglomerate dust grains. J. Quant. Spectrosc. Radiat. Transf. 100, 373-381 (2006).

22. Ishizuka, S., Kimura, Y. \& Sakon, I. In situ infrared measurements of freeflying silicate during condensation in the laboratory. Astrophys. J. 803, 88 (2015).

23. Ishizuka, S., Kimura, Y. \& Yamazaki, T. In situ FT-IR study on the homogeneous nucleation of nanoparticles of titanium oxides from highly supersaturated vapor. J. Cryst. Growth 450, 168-173 (2016).

24. Kimura, Y. et al. Nucleation processes of cosmic dust investigated by microgravity experiments using an airplane. Int. J. Microgravity Sci. Appl. 35, 350305 (2018)

25. Takigawa et al. Morphology and crystal structures of solar and presolar $\mathrm{Al}_{2} \mathrm{O}_{3}$ in unequilibrated ordinary chondrites. Geochim. Cosmochim. Acta 124, 309-327 (2014).

26. Tavakoli, A. H. et al. Amorphous alumina nanoparticles: structure, surface energy, and thermodynamic phase stability. J. Phys. Chem. C 117, 17123-17130 (2013)

27. McHale, J. M., Auroux, A., Perrotta, A. J. \& Navrotsky, A. Surface energies and thermodynamic phase stability in nanocrystalline aluminas. Science $\mathbf{2 7 7}$, 788-791 (1997).

28. Landron et al. Liquid alumina: detailed atomic coordination determined from neutron diffraction data using empirical potential structure refinement. Phys. Rev. Lett. 86, 4839-4842 (2001).

29. Ansell, S. et al. Structure of liquid aluminum oxide. Phys. Rev. Lett. 78, 464-466 (1997)

30. Skinner, L. B. et al. Joint diffraction and modeling approach to the structure of liquid alumina. Phys. Rev. B Condens. Matter Mater. Phys. 87, 53-58 (2013).

31. Kovarik, L. et al. Structure of $\delta$-alumina: toward the atomic level understanding of transition alumina phases. J. Phys. Chem. C 118, 18051-18058 (2014)

32. Kovarik, L. et al. Unraveling the origin of structural disorder in high temperature transition $\mathrm{Al}_{2} \mathrm{O}_{3}$ : structure of $\theta-\mathrm{Al}_{2} \mathrm{O}_{3}$. Chem. Mater. 27, 7042-7049 (2015)

33. Greenhouse, M. A. The JWST science instrument payload: mission context and status. Proc. SPIE 9904, 990406 (2016).

34. Molster, F. \& Kemper, C. Crystalline silicates. Space Sci. Rev. 119, 3-28 (2005).

35. Nakagawa, T. et al. The next-generation infrared astronomy mission SPICA under the new framework. Proc. SPIE 9143, 914311 (2014).

\section{Acknowledgements}

We thank N. Ishii and all project members of the sounding rocket S-520 30 experiment of ISAS, JAXA, Japan, Y. Ikeda of Photocoding for calculating the optical path, and $\mathrm{H}$. Nakagawa of Crystal Optics for manufacturing the mirrors. This work was partly supported by a Grant-in-Aid for Young Scientists (18K13598) and a Grant-in-Aid for Scientific Research (S) from KAKENHI (15H05731). We are indebted to Bruce T. Draine and Piotr J. Flatau who made their DDA code publicly available.

\section{Author contributions}

S.I. conducted the ground-based experiments. S.I., Y.K., I.S., T.Y., and Y.I. prepared the experimental system. S.I. and Y.K. performed the experiment, interpreted the data, and 
co-wrote the paper. Y.K. designed the project. S.T. and Y.I. prepared the interface between the experimental system and sounding rocket. H.K. calculated the IR spectra.

\section{Additional information}

Supplementary Information accompanies this paper at https://doi.org/10.1038/s41467018-06359-y.

Competing interests: The authors declare no competing interests.

Reprints and permission information is available online at http://npg.nature.com/ reprintsandpermissions/

Publisher's note: Springer Nature remains neutral with regard to jurisdictional claims in published maps and institutional affiliations. (c) (i) Open Access This article is licensed under a Creative Commons Attribution 4.0 International License, which permits use, sharing, adaptation, distribution and reproduction in any medium or format, as long as you give appropriate credit to the original author(s) and the source, provide a link to the Creative Commons license, and indicate if changes were made. The images or other third party material in this article are included in the article's Creative Commons license, unless indicated otherwise in a credit line to the material. If material is not included in the article's Creative Commons license and your intended use is not permitted by statutory regulation or exceeds the permitted use, you will need to obtain permission directly from the copyright holder. To view a copy of this license, visit http://creativecommons.org/ licenses/by/4.0/.

(C) The Author(s) 2018 